\title{
Selected Extended Papers of ITP 2016: Preface
}

\author{
Jasmin Christian Blanchette ${ }^{1}$ - Stephan Merz ${ }^{2}$
}

Received: 30 May 2018 / Accepted: 1 June 2018 / Published online: 12 June 2018

(C) Springer Nature B.V. 2018

Interactive Theorem Proving (ITP) is the premier international conference devoted to interactive theorem proving and related subjects, ranging from theoretical foundations to implementation aspects and applications in program verification, security, and the formalization of mathematics. The seventh edition of ITP took place in August 2016 at the Inria research center in Nancy, France. Its proceedings appeared as volume 9807 of Lecture Notes in Computer Science, published by Springer.

The present issue of the Journal of Automated Reasoning contains six articles based on papers presented at ITP 2016. The authors were invited to submit revised and extended versions of their papers, and these were subject to a stringent peer-reviewing process. The articles explore a broad range of applications of interactive theorem proving in mathematics and computer science. Taken together, they witness the current state of the art of the field.

- Chan and Norrish present an elegant and elementary proof of the fact that $2^{n}$ is a lower bound for the least common multiple of the natural numbers between 1 and $n+1$. This property is instrumental for showing that primality of integers can be decided in polynomial time. The proof presented here is purely number-theoretic and is based on manipulations of the Leibniz harmonic triangle. It has been formalized in HOL4.

- Grégoire and Chlipala introduce a mathematical framework, supported by an implementation in Coq, for the verification of sequential and distributed stencil algorithms. These algorithms repeatedly update elements of arrays according to fixed patterns, and they are ubiquitous in scientific computing. The library is intended for proving that accesses to array elements, even in optimized implementations, respect dependencies expressed by the stencil.

Stephan Merz

stephan.merz@inria.fr

Jasmin Christian Blanchette

j.c.blanchette@vu.nl

1 Vrije Universiteit Amsterdam, Amsterdam, The Netherlands

2 University of Lorraine, CNRS, Inria, LORIA, Nancy, France 
- Immler and Traut provide a library, implemented in Isabelle/HOL, for reasoning about the flow of ordinary differential equations (ODEs) - i.e., the solution of the ODE as a function of initial conditions, together with the (maximal) interval for which the solution exists. Besides developing a theory of properties of flows and Poincaré maps, they demonstrate that the library can be used to compute bounds on the solutions of concrete equations.

- Kunčar and Popescu extend the standard mechanism in proof assistants based on higherorder logics for introducing a new type isomorphic to a given nonempty set so that it can be applied even within a local proof context. This extension allows users to easily convert between set-based and type-based formulations of theorems. Their technique has been implemented within Isabelle/HOL, taking into account specific concerns related to axiomatic type classes.

- Lammich and Sefidgar formalize classical algorithms for computing the maximum flow in a network. Starting from a correctness proof of the generic Ford-Fulkerson algorithm, efficient implementations of the algorithms due to Edmonds and Karp and of the push-relabel algorithm designed by Goldberg and Tarjan are derived within a refinement framework implemented in Isabelle/HOL.

- Mahboubi, Melquiond, and Sibut-Pinote propose an efficient method for computing and proving bounds on certain definite integrals inside the Coq proof assistant. They extend their method to handle improper integrals-i.e., limits of definite integrals where bounds tend towards $+\infty$ or 0 when 0 is a singular point of the function to be integrated. The use of the method is demonstrated by comparing it to standard implementations on cases where the latter produce wrong results.

We are grateful to the editor-in-chief, Tobias Nipkow, and to the editorial office of Springer for the excellent collaboration in producing this special issue. We thank the authors for their diligence in revising the papers presented at ITP 2016, ensuring that the articles published here present significant additional contributions. The anonymous reviewers did a wonderful job in providing timely, in-depth reports that have contributed substantially to the quality of this issue.

Amsterdam and Nancy, May 2018

Jasmin Blanchette and Stephan Merz 